\title{
Peculiarities of nonadditive changes in conductivity of nano-PDLC under influence of magnetite and single-wall carbon nanotubes
}

\author{
P. Kopčanský ${ }^{1}$, M. Timko ${ }^{1}$, Z. Mitrova ${ }^{1}$, N. Tomašovičová ${ }^{1}$, L. Tomčo ${ }^{1}$, O.V. Kovalchuk ${ }^{2,3}$, \\ T.M. Kovalchuk ${ }^{4}$, I.V. Oleinikova ${ }^{2}$, A.I. Lad ${ }^{2}$, I.P. Studenyak ${ }^{5}$ \\ ${ }^{I}$ Institute of Experimental Physics, Slovak Academy of Sciences, \\ 47, Watsonova str., 04001 Košice, Slovak Republic \\ ${ }^{2}$ Kyiv National University of Technologies and Design, \\ 2, Nemirovich-Danchenko str., 01011 Kyiv, Ukraine \\ E-mail:akoval@knutd.com.ua \\ ${ }^{3}$ Institute of Physics, National Academy of Sciences of Ukraine, \\ 46, prospect Nauky, 03028 Kyiv, Ukraine \\ ${ }^{4}$ V. Lashkaryov Institute of Semiconductor Physics, NAS of Ukraine, \\ 41, prospect Nauky, 03028 Kyiv, Ukraine \\ ${ }^{5}$ Uzhgorod National University, 46, Pidhirna str., 88000 Uzhgorod, Ukraine
}

\begin{abstract}
Studied in this work has been the effect of nanoparticles - magnetite and single-wall carbon nanotubes - separately and together on the conductivity of nematic liquid crystal 6CHBT dispersed in polyvinyl alcohol. Morphology of these films was analyzed using an electron microscope. When using selected technologyof homogenizing the mixture components, there takes place formation of liquid crystals dispersed in the polymer matrix with the average sizes of liquid-crystal droplets close to $500 \mathrm{~nm}$ (nanoPDLC). It has been found that simultaneous introduction of magnetite and nanotubes results in lowering the conductivity of nano-PDLC as compared to the total conductivity of nano-PDLC with each kind of nanoparticles separately. It has been suggested that the main mechanism of this effect lies in formation of deep centers for electron capture by complexes with different types of nanoparticles, which leads to a decrease in electronic conductivity through the polymer matrix.
\end{abstract}

Keywords: magnetic nanoparticle, single-wall carbon nanotube, permittivity, ion component of conductivity, electron component of conductivity, polymer matrix.

Manuscript received 11.02.14; revised version received 14.05.14; accepted for publication 29.10.14; published online 10.11.14.

\section{Introduction}

An important place in up-to-date scientific works belongs to studies of the effect of nanoparticles on properties of various materials, including liquid crystals, in order to obtain compositions with new functional properties. In the most works, one type of nanoparticles is used. But not always, using only one type of nanoparticles as additives provides preparation of material with desired properties.
When introducing nanoparticles of several different types into a matrix even at low concentrations, it is not always possible to predict the effect of nanoparticles together, if being based on data about the impact of each type of nanoparticles separately. In the work [1], we studied the additive action of nanoparticles - magnetic nanoparticles $(\mathrm{MN})$ and single-wall carbon nanotubes (SWNT) - on properties of homogeneous liquid crystal. It was shown that introducing MN and SWNT with the concentration $0.02 \mathrm{wt} . \%$ for each nanoparticle type into 
liquid crystal leads approximately to the 20 -fold increase in conductivity, while each individual component separately increases the LC conductivity no more than doubly. One of the mechanisms of this effect may be formation of complexes of nanoparticles that contribute to formation of additional charge carriers. The efficiency of this process can be essentially influenced by the size effect (the value of LC volume), which can be achieved by dispersing the LC molecules in the polymer matrix (PDLC cell).

Partly, we solved the problem of the influence of size effect on nonadditivity of action of magnetite and nanotubes on the LC conductivity in the work [2] where PDLC cells with the sizes of nematic droplets $8 \ldots 10 \mu \mathrm{m}$ were studied. It was found that for these sizes the effect of nonadditive action of carbon nanotubes and magnetite on the composite conductivity is lower, but still quite significant. That's why, the purpose of this work was to investigate the possibility of nonadditive action of nanoparticles on the LC conductivity for smaller LC droplet sizes than in [2].

\section{Materials and methods of research}

The samples of PDLCs were prepared using the following method. Liquid crystal 6CHBT of amount $0.05 \mathrm{ml}$ was added to $5 \mathrm{ml}$ of $10 \%$ polyvinyl alcohol (PVA). The samples was sonicated with an ultrasonic oscillator (Branson model 450, $20 \mathrm{kHz}, 280 \mathrm{~W}$ ) for 1 min. A creamy white emulsion was obtained. A thin bead was placed on a slide. After water evaporation, we got a thin film. The sample thickness was $50 \mu \mathrm{m}$. This technique was also used for preparation of the PDLC films doped with various types of magnetic particles. Into PDLC, we entered MN of spherical shape with the average diameter $5 \mathrm{~nm}$ and SWNT with the length $1000 \mathrm{~nm}$ and the diameter $2 \mathrm{~nm}$. Samples with the nanoparticle concentrations $0.01,0.1$ and $0.2 \mathrm{wt} . \%$ were prepared. Where we injected $\mathrm{MN}$ and SWNT, the concentration of each type nanoparticles was the same.

As in [2], morphology of the obtained films was studied using a scanning electron microscope JSM-35 (Japan) at the voltage $35 \mathrm{keV}$ that accelerates the electron beam. Before performing the study, we deposited a thin layer of graphite on the film surface. So, there were minimized effects that were caused by charging the surface. All the obtained images were recorded using a digital camera and then analyzed.

PDLC were deposited on glass plates coated with ITO conductive layer. After drying the solvent, another glass plate covered with a ITO layer was pressed to the free surface of PDLC. To maintain good electrical contact, we deposited a thin LC layer on the electrode before pressing. The sandwich cell required for dielectric measurements was formed in this manner. Since the PDLC film took only the central part of the sample, using the interference method at the edges of the electrodes we measured the distance between the electrodes and consequently the cell thickness.
Measurements of the thickness were performed in different places of the sample. It made it possible to check the uniformity of pressing the top electrode.

Dielectric properties of the samples were studied within the frequency range $10^{-1} \ldots 10^{6} \mathrm{~Hz}$ at the temperature $293 \mathrm{~K}$ by using the oscilloscopic method [3]. The amplitude of the measuring signal with the triangular shape was $0.25 \mathrm{~V}$.

\section{Results and analysis}

\subsection{Morphology of films}

Analysis of morphology of PDLC films showed that, when selected technology of homogenization was used, the sizes of LC impurities in the studied PDLC did not exceed $1 \mathrm{~mm}$ and the average one was $500 \mathrm{~nm}$. Therefore, the obtained dispersions of liquid crystal were called nano-PDLC. The small sizes of LC droplets were confirmed studying the transmission of nano-PDLC in the visible spectral range.

Being based on the analysis of the images of nanoPDLC, we ascertained that morphology of nano-PDLC does not substantially change in dependence on the type and concentration of nanoparticles. Quantitative estimations of the nanoparticle diameter could not be performed because the edges of LC nano-impurities are blurred. It is known from [4] that after the phase separation, when preparing PDLC, clear boundaries between the LC droplets and polymer matrix are not observed. There is always an intermediate layer consisting of a mixture of monomers of polymer and LC molecules (at the molecular level). The thickness of this layer is about $1 \mathrm{~mm}$, which is comparable with the sizes of LC droplets in the case of nano-PDLC.

\subsection{Frequency dependence of the permittivity and conductivity of nano-PDLC with nanoparticles}

Since we have analyzed the frequency dependence of the permittivity $\varepsilon^{\prime}$ and conductivity $\sigma$ of nano-PDLC with $\mathrm{MN}$ in detail in the work [5], let us consider in detail the similar properties of nano-PDLC with SWNT and nanoPDLC with SWNT and MN together.

Fig. 1 shows spectra of permittivity $\varepsilon^{\prime}$ : nanoPDLC (1), nano-PDLC + 0.01 wt.\% SWNT (2), nanoPDLC + 0.1 wt. $\%$ SWNT (3) and nano-PDLC + 0.2 wt. $\%$ SWNT (4).

As it was shown in [6], significant values of $\varepsilon^{\prime}$ (especially at low frequencies) are caused by effect of the near-electrode processes. As seen from Fig. 1, the greatest impact of SWNT on the $\varepsilon^{\prime}$ value is observed in the range of frequencies 1 to $10^{3} \mathrm{~Hz}$. At the lowest frequencies $f$ as well as for $f>10^{3} \mathrm{~Hz}$, SWNT impact on the $\varepsilon^{\prime}$ value is much lower. The part of the dielectric spectrum for $f>10^{3} \mathrm{~Hz}$ displays bulk properties of the samples. Therefore, we can conclude that at the indicated concentrations, SWNT does not significantly change the $\varepsilon^{\prime}$ value of the nano-PDLC bulk. 


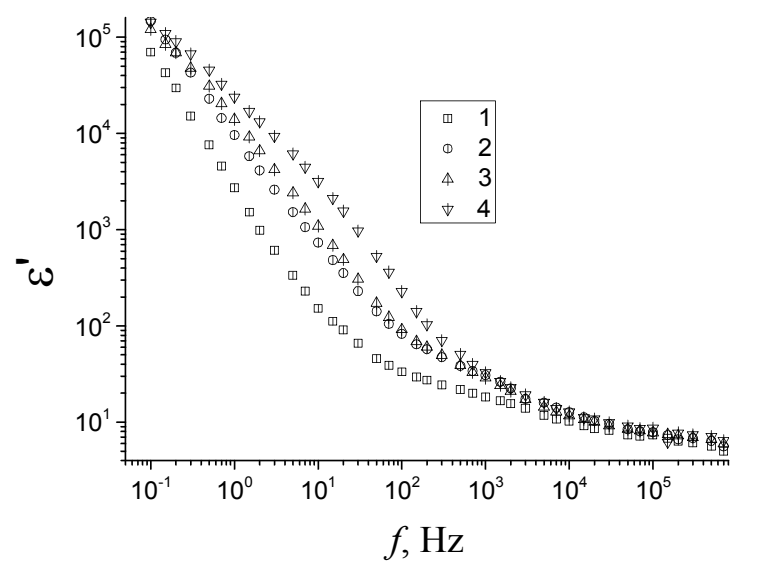

Fig. 1. Frequency dependences of permittivity $\varepsilon^{\prime}$ : nanoPDLC (1), nano-PDLC + 0.01 wt.\% SWNT (2), nano-PDLC + 0.1 wt.\% SWNT (3), and nano-PDLC + 0.2 wt.\% SWNT (4). The sample thickness is $50 \pm 5 \mu \mathrm{m}$. The temperature $273 \mathrm{~K}$.

The decrease of the influence of SWNT on the $\varepsilon^{\prime}$ value of nano-PDLC at the frequencies $f<1 \mathrm{~Hz}$ can be explained by taking into account the structure of nearelectrode layer. As it is known, this layer consists of ordered (closest to the electrode) and diffuse parts. It was shown in [6] that the lower the frequency, the thinner is the part of near-electrode layer, to which the electric field is applied. From this it follows that reducing the impact of SWNT on the $\varepsilon^{\prime}$ value of nanoPDLC at the frequencies $f<1 \mathrm{~Hz}$ corresponds to processes in the ordered part of the near-electrode layer, the thickness of which is smaller than that of SWNT.

Fig. 2 shows the spectra of permittivity $\varepsilon^{\prime}$ : nanoPDLC (1), nano-PDLC + 0.01 wt. $\%$ SWNT + 0.01 wt.\% $\mathrm{MN}(2)$, nano-PDLC + $0.1 \mathrm{wt} . \% \mathrm{SWNT}+0.1 \mathrm{wt} . \% \mathrm{MN}$ (3), and nano-PDLC +0.2 wt.\% SWNT $+0.2 \mathrm{wt} . \%$ MN (4).

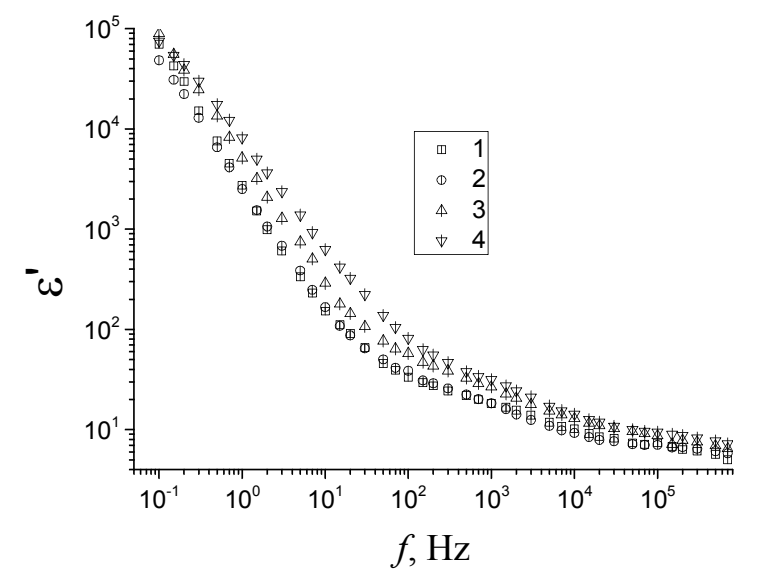

Fig. 2. Frequency dependences of permittivity $\varepsilon^{\prime}$ : nano-PDLC (1), nano-PDLC $+0.01 \mathrm{wt} . \%$ SWNT $+0.01 \mathrm{wt} . \% \mathrm{MN}(2)$, nano-PDLC + 0.1 wt. \% SWNT + 0.1 wt. $\%$ MN (3), and nanoPDLC +0.2 wt. $\%$ SWNT +0.2 wt. $\%$ MN (4). The sample thickness is $50 \pm 5 \mu \mathrm{m}$. The temperature $273 \mathrm{~K}$.
From the comparison of Figs 1 and 2, it can be concluded that the above mentioned regularities of influence of SWNT on the value $\varepsilon^{\prime}$ of nano-PDLC are also kept when adding MN. However, the effect of SWNT separately on the $\varepsilon^{\prime}$ value of nano-PDLC is greater than that of SWNT and MN together. This fact is very important to explain the impact of nanoparticles on the conductivity of nano-PDLC.

Fig. 3 shows the frequency dependence of the conductivity $\sigma$ : nano-PDLC (1), nano-PDLC + 0.01 wt. $\%$ SWNT (2), nano-PDLC + 0.1 wt. $\%$ SWNT (3), and nano-PDLC + 0.2 wt.\% SWNT (4).

As it follows from the analysis of the data, with few exception of nano-PDLC without nanoparticles, there are no parts on the frequency dependences of conductivity, where the $\sigma$ value does not depend on frequency. In all the samples of micro-PDLC, we observed spectral regions where the conductivity is independent of frequency, and we explained this effect by ionic conductivity through the LC drops [2]. Due to large sizes, these drops either "locked" electrodes in their location (it was clearly visible in the photos) or the distance between the drops was sufficient for the passage of ions.

It follows from the analysis of Fig. 1 (curve 1) that for the frequencies from 1 to $10^{3} \mathrm{~Hz}$, the conductivity is a power function of the frequency with a small exponent.

Summarizing, we can conclude that unlike microPDLC, in nano-PDLC the purely ionic conductivity is not observed. From this conclusion does not follow that the ionic conductivity is excluded from the process of charge transfer through the sample. However, contribution of the ionic conductivity is not essential, because it is limited by the conductivity of the polymer parts between LC drops.

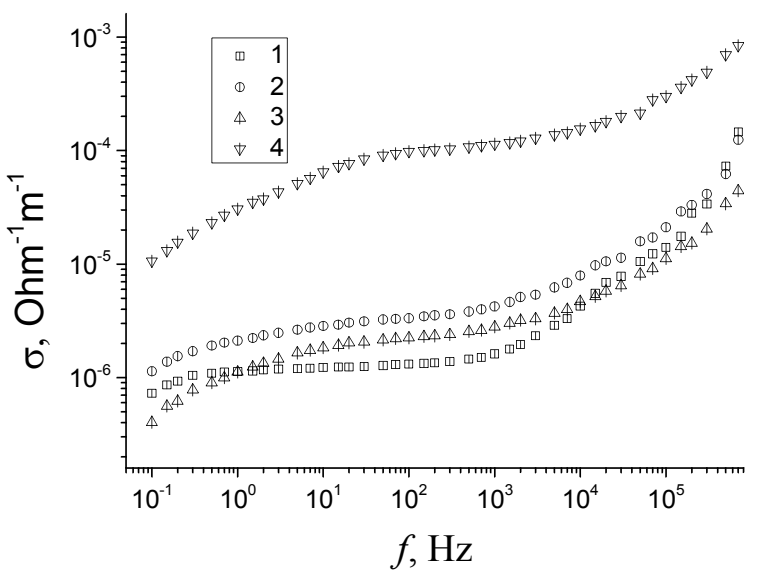

Fig. 3. Frequency dependences of conductivity $\sigma$ : nano-PDLC (1), nano-PDLC + $0.01 \mathrm{wt} \%$ SWNT (2), nano-PDLC + $0.1 \mathrm{wt} . \%$ SWNT (3), and nano-PDLC + $0.2 \mathrm{wt} . \%$ SWNT (4). The sample thickness is $50 \pm 5 \mu \mathrm{m}$. The temperature $273 \mathrm{~K}$. 
It is the significant influence of conductivity electron components in nano-PDLC, as compared to micro-PDLC, that can explain the sharp increase in conductivity for the samples with the concentration 0.2 wt.\% SWNT (Fig. 3, curve 4) almost over the whole frequency range. This effect can be explained by creation of a percolation lattice between SWNT [7, 8]. Intermediate links between SWNT is nano-PDLC with electron type conductivity.

To determine features of this percolation, it is reasonable to study how the conductivity of nano-PDLC will change when $\mathrm{MN}$ and SWNT are added together to nano-PDLC. Fig. 4 shows the frequency dependence of $\sigma$ conductivity: nano-PDLC (1), nano-PDLC + 0.01 wt. $\%$ SWNT +0.01 wt. $\%$ MN (2), nano-PDLC + 0.1 wt. $\%$ SWNT + 0.1 wt. $\%$ MN (3) and nano-PDLC + 0.2 wt. $\%$ SWNT + 0.2 wt. $\% \mathrm{MN}(4)$.

The most important conclusion to be drawn from Fig. 4 is the lack of a sharp increase in the conductivity for the sample nano-PDLC $+0.2 \mathrm{wt} \%$ SWNT + 0.2 wt.\% MN (curve 4). That is adding to the sample nano-PDLC $+0.2 \mathrm{wt} \%$ SWNT of small amount of $0.2 \mathrm{wt} . \% \mathrm{MN}$ leads to the fact that in contrast to the data shown in Fig. 3 there is no abrupt change in the conductivity at the nanotube concentration $0.2 \mathrm{wt} . \%$. The reason for this effect may be creation of complexes of nanoparticles with SWNT and MN. These complexes may reduce the efficiency of formation of percolation lattice and thus increase the concentration of nanoparticles in the case of percolation transition.

\subsection{Nonadditive effect of magnetic nanoparticles, carbon nanotubes (separately and together) on the conductivity of nano-PDLC}

After analyzing the properties of the samples under study, let us consider the main problem of this work - to determine features of nonadditive action of $\mathrm{MN}$ and SWNT on the conductivity of nano-PDLC. To characterize the difference between the conductivity of the samples with two types of nanoparticles and the conductivity of samples with each particle type separately, in the work [2] we proposed to use the coefficient of nonadditivity. To calculate this coefficient, we used the following relation

$k_{\text {nonad }}=\frac{\sigma_{12}}{\sigma_{1}+\sigma_{2}}$,

where $\sigma_{12}$ is the conductivity value when entering $\mathrm{MN}$ and SWNT together into nano-PDLC, $\sigma_{1}$ and $\sigma_{2}$ are the values of conductivity under the action of each type of nanoparticles separately.

Fig. 5 shows the frequency dependence of $k_{\text {nonad }}$ for three different concentrations of nanoparticles: 0.01 wt.\% (1), 0.1 wt.\% (2), and 0.2 wt.\% (3).

The main conclusion following from the analysis of Fig. 5 can be summarized as follows: regardless of the chosen concentrations of nanoparticles, the conductivity of nano-PDLC with two types of nanoparticles is lower than that with combined effect of each nanoparticle separately (the value of $k_{\text {nonad }}$ for all the selected concentrations is less than unity). This property fundamentally distinguishes the effect of nanoparticles on the conductivity of nano-PDLC from the action of the same type of nanoparticles on the conductivity of homogeneous liquid crystal and micro-PDLC.

It is caused by the fact (as already noted above) that the ionic conductivity is not revealed in a "pure" form in nano-PDLC. Therefore, there is no appropriate mechanisms that would lead to an increase in the $k_{\text {nonad }}$ value. In the case of electron transfer, morphology of PDLC films will essentially influence on the presence of deep levels for electron capture. Analysis of the influence of type and concentration nanoparticles (within the ranges that were taken for these researches) showed that morphology of nano-PDLC is not significantly changed under the influence of nanoparticles. Therefore, we can predict that the main mechanism of small values of $k_{\text {nonad }}$ in the case of nano-PDLC is creation of deep levels for capturing carriers by the above nanoparticles of two types.

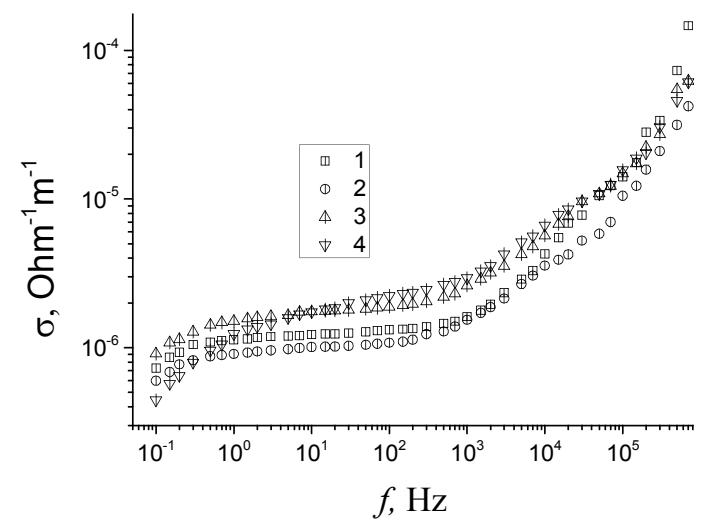

Fig. 4. Frequency dependences of conductivity $\sigma$ : nano-PDLC (1), nano-PDLC +0.01 wt. $\%$ SWNT +0.01 wt.\% MN(2), nano-PDLC + 0.1 wt. $\%$ SWNT $+0.1 \mathrm{wt} . \% \mathrm{MN}(3)$, and nanoPDLC +0.2 wt. $\%$ SWNT +0.2 wt. $\%$ MN (4). The sample thickness is $50 \pm 5 \mu \mathrm{m}$. The temperature $273 \mathrm{~K}$.

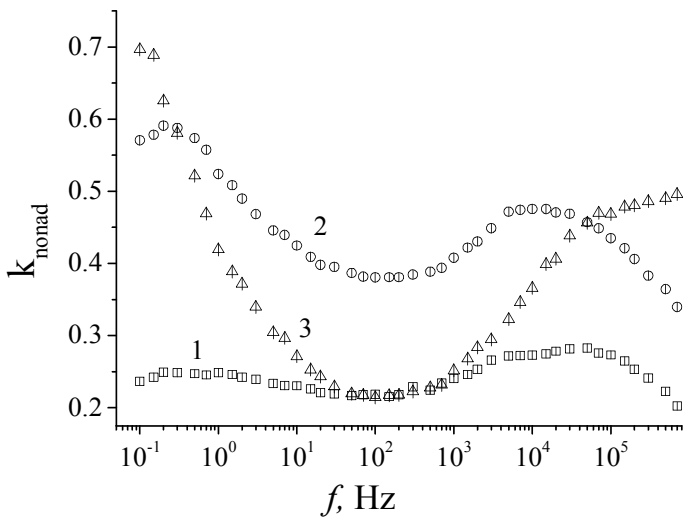

Fig. 5. Frequency dependences of $k_{\text {nonad }}$ of nano-PDLC for three different concentrations of nanoparticles: $0.01 \mathrm{wt} . \%(1)$, 0.1 wt. $\%$ (2), and $0.2 \mathrm{wt} . \%$ (3). The sample thickness is $50 \pm 5 \mu \mathrm{m}$. The temperature $273 \mathrm{~K}$. 
In the framework of the proposed mechanism that explains the reasons of small values of $k_{\text {nonad }}$ in the case of nano-PDLC, it is useful to analyze the frequency dependence of nano-PDLC for different concentrations of nanoparticles (Fig. 5). As follows from the analysis of this figure, on average (within the frequency range) the samples with the lowest concentrations of the studied nanoparticles $(0.01 \mathrm{wt} . \%)$ have the smallest $k_{\text {nonad }}$ value. One may assume that it is for this concentration of nanoparticles, the deep levels of electron capture, which are caused by complexes of two different types of nanoparticles, most efficiently influence on the transport of charge carriers.

In the case of increasing the nanoparticle concentration, besides the centers of electron capture, the additional channels of electron transfer through polymer may also arise in nano-PDLC. The efficiency of increasing the charge carrier concentration may be higher than that of increasing the concentration of the centres for electron capture. This may be the reason for the increase of the $k_{\text {nonad }}$ value with the concentration of nanoparticles (curves 2 and 3 in Fig. 5).

\section{Conclusions}

Thus it is shown that, as in micro-PDLC, introduction of magnetic nanoparticles and nanotubes with the concentrations less than $0.2 \mathrm{wt} . \%$ separately and together into nano-PDLC cannot significantly change morphology of the films.

In contrast to the homogeneous liquid crystal and micro-PDLC, the conductivity of nano-PDLC with MN and SWNT is less than the total effect of each nanoparticle separately. The main factors for this effect are: (i) lack of ionic component of conductivity through nano-PDLC; (ii) formation of deep centers for capture of electrons by complexes with different types of nanoparticles. It has been shown that the second factor operates most efficiently, when the chosen concentration of nanoparticles is the lowest one ( $0.01 \mathrm{wt} . \%)$.

Formation of complexes between MN and SWNT is the reason for increasing the concentration of nanoparticles in the case of percolation transition, when MN and SWNT act jointly on the conductivity of nanoPDLC with respect to the effect only of the nanotubes.

\section{References}

1. P. Kopčanský, O.V. Kovalchuk, N. Tomašovičová et al., Nonadditive changes in the conductivity of nematic liquid crystal when introducing singlewalled carbon nanotubes and magnetite // The $13^{\text {th }}$ Small Triangle Meeting on Theoretical Physics. Institute of Experimental Physics, Kosice, Slovakia, p. $72-79$ (2012).

2. P. Kopčanský, M. Timko, Z. Mitrova, V. Zavisova, M. Koneracká, N. Tomašovičová, L. Tomčo, O.V. Kovalchuk, V.M. Bykov, T.M. Kovalchuk, Nonadditive changes in conductivity of micro PDLC under the influence of carbon nanotubes and magnetic nanoparticles // Semiconductor Physics, Quantum Electronics and Optoelectronics, 16(4), p. 374-378 (2013).

3. A.J. Twarowski, A.C. Albrecht, Depletion layer in organic films: Low frequency measurements in polycrystalline tetracene // J. Chem. Phys. 20, p. 2255 (1979).

4. H. Stark, Physics of colloidal dispersions in nematic liquid crystals // Phys. Repts. 351(6), p. 387-474 (2001).

5. P. Kopčanský, M. Timko, Z. Mitrova, V. Zavisova, M. Koneracká, N. Tomašovičová, L. Tomčo, O.V. Kovalchuk, V.M. Bykov, T.M. Kovalchuk, A.I. Lad, Effect of magnetic nanoparticles with various geometrical shapes on morphology and dielectric properties of nanodispersions of nematic liquid crystal in polymer matrix // Semiconductor Physics, Quantum Electronics and Optoelectronics, 16(3), p. 253-258 (2013).

6. A.V. Koval'chuk, Relaxation processes and charge transport across liquid crystal-electrode interface // J. Phys.: Condens. Matter, 13, p. 10333 (2001).

7. A.I. Goncharuk, N.I. Lebovka, L.N. Lisetski, and S.S. Minenko, Aggregation, percolation and phase transitions in nematic liquid crystal EBBA doped with carbon nanotubes // J. Phys. D: Appl. Phys. 42, 165411 (2009).

8. A. Koval'chuk, L. Dolgov, O. Yaroshchuk, Dielectric studies of dispersions of carbon nanotubes in liquid crystals 5CB // Semiconductor Physics, Quantum Electronics \& Optoelectronics, 11, p. 337 (2008). 